\title{
PALS investigations of matrix Vycor glass doped with molecules of luminescent dye and silver nanoparticles. Discrepancies from the ETE model
}

Marek Gorgol,
Bożena Jasińska,
Renata Reisfeld

Abstract. A thermal stability of three materials: undoped reference Vycor glass, glass filled with ROT-305 red dye, and silver nanoparticles was investigated by positron annihilation lifetime spectroscopy (PALS) in a broad temperature range (from 93 to $473 \mathrm{~K}$ ). The attempt of pore size calculations from the ortho-positronium lifetime data was performed using the extended Tao-Eldrup (ETE) model. Below room temperature, a significant decrease in lifetime values of the longest-lived component was found for all the samples. This effect could not be explained by thermal shrinkage of the material and is probably caused by interaction of $o$-Ps with a Vycor glass matrix. The greatest discrepancy from the ETE model predictions was observed for the reference glass. Doping the base material with dye molecules and silver nanoparticles resulted in similar small decrease in this discrepancy. After reheating the samples to the room temperature, the PALS components returned to the initial values. In the temperature range of 293-473 K, quite good agreement between PALS results and the ETE model predictions was observed for the reference glass and the glass incorporated with dye molecules. The observed small discrepancy in this range could possibly be partly explained by thermal expansion of the material. For the glass doped with silver nanoparticles, a significant change in PALS parameters was observed in the temperature range from 403 to $473 \mathrm{~K}$.

Key words: positron annihilation lifetime spectroscopy (PALS) $\bullet$ porous glass $\bullet$ lumogen dye ROT-305 $\bullet$ silver nanoparticles $•$ Vycor glass

\author{
M. Gorgol ${ }^{\bowtie}$, B. Jasińska \\ Department of Nuclear Methods, \\ Institute of Physics, \\ Maria Curie-Skłodowska University, \\ 1 M. Curie-Skłodowskiej Sq., 20-030 Lublin, Poland, \\ Tel.: +48 81537 6123, Fax: +48 815376191 , \\ E-mail: marek.gorgol@gmail.com \\ R. Reisfeld \\ Chemistry Institute, \\ Hebrew University of Jerusalem, \\ Givat-Ram, Jerusalem 91904, Israel
}

Received: 8 July 2015

Accepted: 28 August 2015

\section{Introduction}

Silver nanoparticles (AgNPs) have become a very interesting material because of their possible practical applications, e.g. in medicine. On the other hand, organic dyes are commonly examined as optically active materials for solar concentrators [1] or hybrid light-emitting diodes (LEDs) [2]. However, devices based on such materials show low photon-to-electron conversion efficiency of large plates and low photostability [3]. This could be changed by incorporation them into an inorganic matrix. The etched borosilicate porous glass (Vycor glass) is very popular because of its high availability and a simple manufacturing process. Therefore, it was proposed as a host material for newly synthesized composite materials containing either AgNPs or organic dye molecules. Conducting research of such materials, especially their stability because of changes in external condition, such as thermal treatment, is crucial.

Positron annihilation lifetime spectroscopy (PALS) can give information about the porous structure of studied materials and its dependence on various factors, e.g. pressure [4] or temperature [5-7]. PALS is also suitable for investigation of pore filling of host material with guest molecules 
[8]. In this technique, the probe used for pore size characterization is positronium (bound, quasi-stable state of a positron and an electron). The mean ortho-positronium ( $o$-Ps) lifetime is connected to free volume size, and this relation is given for big pores $(R>$ $1 \mathrm{~nm}$ ) by extended Tao-Eldrup (ETE) model [9]. In this model, a pore is approximated by the potential well of respective shape, where ortho-positronium can occupy either ground or excited energy levels. Owing to population of states, the model predicts the decrease in $o$-Ps lifetime value with the increase in temperature, even if the size of the pores are not changed, what was experimentally confirmed [6].

\section{Material and experimental}

The examined mesoporous Vycor glass was obtained with the use of procedure described in Ref. [10]. In the reference material, the average pore sizes presented a wide distribution from 15 to $25 \mathrm{~nm}$ [11], as determined by liquid nitrogen (LN) sorption and small-angle X-ray scattering (SAXS). Basic parameters for the reference glass obtained by SAXS are presented in Table 1. One part of the samples was incorporated with the commercially available fluorescent dye lumogen red 305 (BASF) with a well-determined particle length of about $10 \mathrm{~nm}$. For comparison, another part of undoped glass was incorporated with silver nanoparticles (AgNPs), obtained using the procedure described in [12]. The average size of nanoparticles was about $10 \mathrm{~nm}$.

The ${ }^{22} \mathrm{Na}$ positron source with the activity of about $180 \mathrm{kBq}$ was placed inside the vacuum chamber between two layers of sample, ensuring that positrons from the source annihilate inside the investigated material. A turbomolecular pump provided pressure of about $10^{-5} \mathrm{~Pa}$ inside a chamber. Each sample was initially degassed for 24 hours at room temperature.

The measurements were performed in a wide range of temperatures using an apparatus consisted of a resistance heater and a Dewar vessel with LN. The temperature was controlled by Shimaden MR-13 PID controller. At the beginning of the experiment, each sample was cooled down from the room temperature to $93 \mathrm{~K}$ and the positron annihilation lifetime (PAL) spectra were collected in the $20 \mathrm{~K}$ steps. Next measurements were performed during heating the sample, as a function of rising temperature. For the clarity of the results presentation, the heating sequence was shifted of $10 \mathrm{~K}$ and the first spectrum was collected at $103 \mathrm{~K}$. The measurements were performed in the steps of $20 \mathrm{~K}$, while the last spectrum was collected at $473 \mathrm{~K}$. Next measurements were made for every $20 \mathrm{~K}$ during cooling down the sample to the room temperature.

Table 1. SAXS data for undoped reference glass

\begin{tabular}{lc}
\hline \multicolumn{1}{c}{ Parameter } & Undoped reference glass \\
\hline Density $\left[\mathrm{g} / \mathrm{cm}^{3}\right]$ & 2.24 \\
Specific surface area $\left[\mathrm{m}^{2} / \mathrm{cm}^{3}\right]$ & 139 \\
Specific surface area $\left[\mathrm{m}^{2} / \mathrm{g}\right]$ & 62 \\
Average pore diameter $[\mathrm{nm}]$ & 19.4 \\
\hline
\end{tabular}

A standard fast-slow delayed coincidence spectrometer was used to collect positron lifetime spectra. The quanta were detected by two scintillation detectors equipped with $\mathrm{BaF}_{2}$ scintillators. A time range of time-to-amplitude converter was $2 \mu \mathrm{s}$, which provided ability to do measurements in a whole range of lifetimes up to $142 \mathrm{~ns}$. The resolution curve of the PAL spectrometer was approximated using two Gaussians: one with a full width at half maximum (FWHM) of about 300 ps with the contribution of $80 \%$, and the other with an FWHM of about 700 ps and contribution of $20 \%$. The ratio of positron registration was about $1.7 \times 10^{6}$ counts per hour, and total count number per each spectrum was $5 \times 10^{6}$.

Positron annihilation lifetime spectra were analyzed using LT 9.2 program [13].

\section{Results and discussion}

The LT analyses, with the use of five discrete lifetime components, for undoped reference glass and two types of glass, one doped with red dye ROT-305 and the other one doped with silver nanoparticles, were performed. The components were ascribed to para-positronium (fixed at $125 \mathrm{ps}$ ), free annihilation of $\mathrm{e}^{+}$and $\mathrm{e}^{-}$and three components connected to annihilation of ortho-positronium in three kind of voids, respectively. The spectra collected at room temperature were additionally analyzed using MELT program [14], and the ortho-positronium lifetime distribution for each sample is shown in Fig. 1. The presence of few peaks in the lifetime range of $0.7-4$ ns could be probably caused by fitting procedures implemented into MELT program.

In the reference glass, only mesopores are large enough to be filled by guest molecules. Therefore, the most interesting is the lifetime of the longest-lived component, which describes $o$-Ps annihilation inside this type of pores. The results of $\tau_{5}$ values, as a function of temperature are shown in Fig. 2, while exact values for a few selected points of particular interest are presented in Table 2 .

For undoped reference glass, the $\tau_{5}$ value of $121.9(6)$ ns was found at room temperature using LT

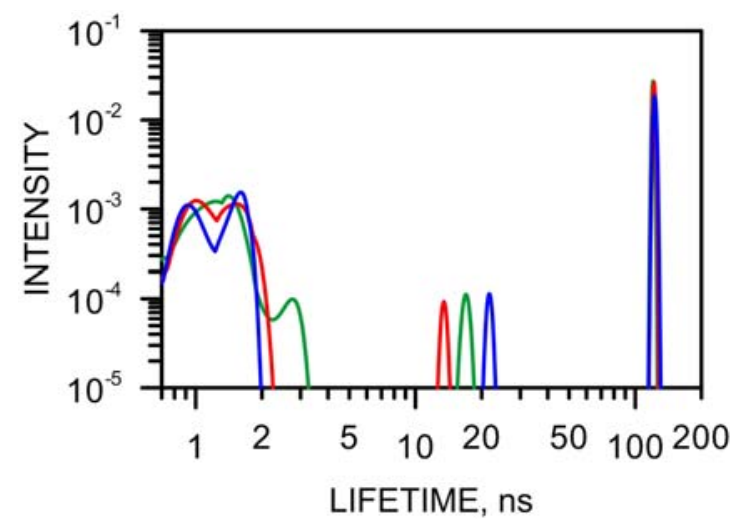

Fig. 1. The $o$-Ps lifetime distribution at room temperature received using MELT procedure for undoped Vycor glass (red line), glass incorporated with ROT-305 red dye (blue line), and glass with silver nanoparticles (green line). 
Table 2. $o$-Ps lifetime values of longest-lived component for undoped, reference glass and samples doped with ROT-305 red dye and silver nanoparticles at few selected temperatures. The column sequence is in agreement with the measurement sequence

\begin{tabular}{lcrrrr}
\hline \multirow{2}{*}{ Sample } & \multicolumn{5}{c}{$\tau_{5}[\mathrm{~ns}]$} \\
\cline { 2 - 6 } & $293 \mathrm{~K}$ & $93 \mathrm{~K}$ & $303 \mathrm{~K}$ & \multicolumn{1}{c}{$473 \mathrm{~K}$} & $293 \mathrm{~K}$ \\
\hline Undoped glass & $121.9(6)$ & $91.7(7)$ & $122.7(5)$ & $118.6(7)$ & $112.2(6)$ \\
Glass + ROT-305 & $122.7(5)$ & $99.6(8)$ & $123.6(7)$ & $118.5(5)$ & $114.4(6)$ \\
Glass + AgNPs & $120.9(6)$ & $102.9(8)$ & $119.5(7)$ & $92.0(5)$ & $102.7(7)$ \\
\hline
\end{tabular}

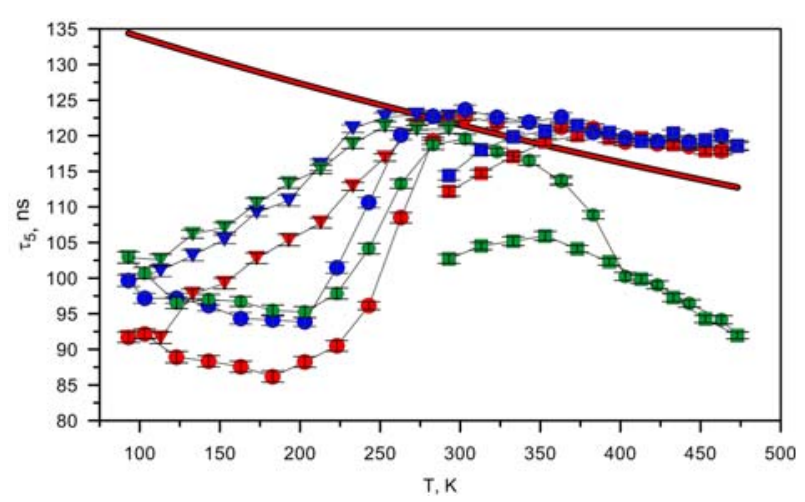

Fig. 2. Temperature dependence of longest-lived component lifetime for undoped Vycor glass (red points), glass incorporated with ROT-305 red dye (blue point), and glass with silver nanoparticles (green points). Triangles are ascribed to cooling the samples down from room temperature to $93 \mathrm{~K}$, circles to heating from 93 to $473 \mathrm{~K}$, and squares to recooling down to the room temperature. Fitted curve shows approximation of the ETE model for reference material.

program. The ETE model was used to evaluate the average mesopore radius and a value of 9.32(3) nm was determined. Because of an elongated pore shape, obtained during etching, a cylindrical potential well was assumed for ETE calculations. During cooling down to $93 \mathrm{~K}$, a great decrease in lifetime values was noticed below $253 \mathrm{~K}$. Similar behavior was previously observed for porous glass [15]. At $93 \mathrm{~K}$, the $\tau_{5}$ reached the value of $91.7(7) \mathrm{ns}$, which would correspond to a pore radius of $2.12(2) \mathrm{nm}$ according to the ETE calculations. Such a big thermal shrinkage of the material is very unreliable, so another explanation of $\tau_{5}$ decrease must be proposed. One possible reason of such an effect is $o$-Ps chemical quenching because of the interaction with residues from the manufacturing process. Further $\tau_{5}$ decrease during heating the sample could be probably the result of a possible time instability of the material and requires additional measurements. However, above $183 \mathrm{~K}$, the $\tau_{5}$ started to increase significantly and returned to its initial value at room temperature. In high temperature range, quite good agreement between experimental and theoretical lifetime values was observed. At $473 \mathrm{~K}$, the difference between two pore radii values (measured and calculated from the ETE model) was 2.81(53) nm and the experimental values were higher than the theoretical ones. This could be partly explained by thermal expansion of the material. However, recooling to the room temperature resulted in a decrease in $\tau_{5}$ value to $112.2(6) \mathrm{ns}$, which is $9.7(8) \mathrm{ns}$ lower than the initial one and needs further examination to be explained. One of possible explanations may come from removing some part of residuals during heating under vacuum.
Doping the base material with ROT-305 molecules did not affect PALS results significantly, in comparison to the reference glass. At room temperature, the $\tau_{5}$ reached 122.7(5) ns. This slight increase in comparison to the base material was discussed more broadly in [11]. A shape of temperature dependence of the longest-lived component lifetime was similar for both samples: the undoped material and the one incorporated with dye molecules. The greatest discrepancy was visible in a low temperature range, for which doping the base material with ROT-305 resulted in decrease of a hysteresis loop of lifetime values. At $93 \mathrm{~K}$, the $\tau_{5}$ reached 99.6(8) ns, which was 7.9(11) ns higher than the corresponding value for the undoped material. The probable interpretation is: 1) deposition of dye molecules on the pore walls inhibited quenching abilities of manufacturing process residues, 2) dye molecules fill the narrower pores. In the high temperature range, no significant influence of doping the base material with dye molecules on temperature dependence of $\tau_{5}$ was observed.

One can expect that incorporation of silver nanoparticles into Vycor reference glass should result in $o$-Ps lifetime decrease because of a high electron density of metallic molecules. However, in the low temperature range, incorporation of nanoparticles changes the $\tau_{5}$ temperature dependence, in comparison to the reference material, in the same way as dye molecules of organic nature. Moreover, this is unusual because of different size of dopants molecules. During heating above $283 \mathrm{~K}, \tau_{5}$ reached lower values than in the case of undoped glass and material composed of glass and red dye at corresponding temperatures. In the high temperature range, a greater decrease in $\tau_{5}$ values was observed, and above $403 \mathrm{~K}$, different shape of the lifetime vs. temperature relationship occurred. This was probably caused by a reorganization of material structure. Additional information can be deduced from physical properties of the sample. Before temperature treatment, the color of sample was brown, which can be considered as coming from nonmetallic molecules of silver oxide. After heating process, the sample incorporated with silver nanoparticles became transparent, which may indicate deoxidation of silver. This could probably lead to a significant $o$-Ps quenching because of an interaction with metallic molecules.

\section{Summary}

PALS measurements were performed to examine the thermal stability of porous Vycor glass and 
its applicability as a host for filling the pores with ROT-305 and AgNPs. At the temperature range from 293 to $473 \mathrm{~K}$, the temperature dependence of $\tau_{5}$ values for reference glass was in quite good agreement with the ETE model predictions. However, the measurements at low temperatures showed the presence of some particles or chemical bonds interacting with ortho-positronium, causing the chemical quenching of it. Moreover, these particles were probably partially removed during heating up to $473 \mathrm{~K}$. Therefore, they may possibly be residues from the manufacturing process.

Filling the pores with dopants caused the decrease in the previously mentioned quenching properties, observed for reference glass. The changes in $\tau_{5}$ values temperature dependences because of filling the pores with ROT-305 and AgNPs are similar at low temperatures, regardless of the type of dopant. This could probably be caused by oxidation of silver nanoparticles, and this process was reversed after heating the sample above $403 \mathrm{~K}$. At higher temperatures, the presence of AgNPs significantly shortened $o$-Ps lifetime values.

Acknowledgments. This work is partly supported by the grant 2013/09/D/ST2/03712 of the National Science Center in Poland.

\section{References}

1. Edelenbosch, O. Y., Fisher, M., Patrignani, L., Sark, W. G. J. H. M., \& van Chatten, A. J. (2013). Luminescent solar concentrators with fiber geometry. Opt. Express, 21(9), A503-A514. DOI: 10.1364/OE.21.00A503.

2. Mosca, M., Caruso, F., Zambito, L., Seminara, B., Macaluso, R., Cali, C., \& Feltin, E. (2013). Warm white LED light by frequency down-conversion of mixed yellow and Red lumogen. Integrated Photonics: Materials, Devices and Applications II, 8767, 0L1-10. DOI: $10.1117 / 12.2017274$.

3. Reisfeld, R., Levchenko, V., Saraidarov, T., Rysiakiewicz-Pasek, E., Baranowski, M., Podhorodecki, A., Misiewicz, J., \& Antropova, T. V. (2012). Steady state and femtosecond spectroscopy of Perylimide Red dye in porous and sol-gel glasses. Chem. Phys. Lett., 546, 171-175. DOI: 10.1016/j.cplett.2012.07.073.

4. Zaleski, R., Goworek, J., Borówka, A., Kierys, A., \& Wiśniewski, M. (2009). Positron porosimetry study of mechanical stability of ordered mesoporous materials. In N. Seaton, F. R. Reinoso, P. Llewellyn, \& S. Kaskel (Eds.), Characterisation of porous solids VIII (pp. 400-407). Cambridge: RSC Publishing. DOI: 10.1039/9781847559418-00400.
5. Fischer, C. G., Weber, M. H., Wang, C. L., McNeil, S. P., \& Lynn, K. (2005). Positronium in low temperature mesoporous films. Phys. Rev. B, 71(18), 180102. DOI: 10.1103/PhysRevB.71.180102.

6. Śniegocka, M., Jasińska, B., Zaleski, R., \& Goworek, T. (2006). Temperature dependence of o-Ps lifetime in some porous media. Deviation from ETE model. Chem. Phys. Lett., 430, 351-354. DOI: 10.1016/j. cplett.2006.09.001.

7. Thraenert, S., Hassan, E. M., Enke, D., Fuerst, D., \& Krause-Rehberg, R. (2007). Verifying the RTE model: ortho-positronium lifetime measurement on controlled pore glasses. Phys. Status Solidi C, 4(10), 3819-3822. DOI: 10.1002/pssc.200675738.

8. Kullman, J., Enke, D., Thraenert, S., Krause-Rehberg, R., \& Beiner, M. (2012). Characterization of pore filling of mesoporous host systems by means of positronium annihilation lifetime spectroscopy (PALS). Opt. Appl., XLII(2), 281-286. DOI: 10.5277/oa120205.

9. Ciesielski, K., Dawidowicz, A. L., Goworek, T., Jasińska, B., \& Wawryszczuk, J. (1998). Positronium lifetimes in porous Vycor glass. Chem. Phys. Lett ., 289(1/2), 41-45. DOI: 10.1016/S00092614(98)00416-3.

10. Vasilevskaya, T. N., \& Antropova, T. V. (2009). Small angle X-ray scattering study of the structure of glassy nanoporous matrices. Phys. Solid State, 51(12), 2537-2545. DOI: 10.1134/S1063783409120178.

11. Reisfeld, R., Jasinska, B., Levchenko, V., Gorgol, M., Saraidarov, T., Popov, I., Antropova, T. V., \& Rysiakiewicz-Pasek, E. (2015). Porous glasses as a host of luminescent materials, their applications and site selective determination. J. Lumines. (in press). DOI: 10.1016/j.jlumin.2015.02.022.

12. Saraidarov, T., Levchenko, V., \& Reisfeld, R. (2010). Synthesis of silver nanoparticles and their stabilization in different sol-gel matrices: Optical and structural characterization. Phys. Status Solidi C, 7(11/12), 2648-2651. DOI: 10.1002/pssc.200983784.

13. Kansy, J. (1996). Microcomputer program for analysis of positron annihilation lifetime spectra. Nucl. Instrum. Methods Phys. Res., Sect. A-Accel. Spectrom. Dect. Assoc. Equip., 374(2), 235-244. DOI: 10.1016/0168-9002(96)00075-7.

14. Shukla, A., Peter, M., \& Hoffmann, L. (1993). Analysis of positron lifetime spectra using quantified maximum entropy and a general linear filter. Nucl. Instrum. Methods Phys. Res. Sect. A-Accel. Spectrom. Dect. Assoc. Equip., 335, 310-317. DOI: 10.1016/0168-9002(93)90286-Q.

15. Kullman, J., Enke, D., Thraenert, S., Krause-Rehberg, R., \& Inayat, A. (2010). Characterization of nanoporous monoliths using nitrogen adsorption and positronium annihilation lifetime spectroscopy. Colloid Surf. A-Physicochem. Eng. Asp., 357, 17-20. DOI: 10.1016/j.colsurfa.2009.09.030. 